\title{
Up-to-date teaching and learning of quantum optics at the beginning of the XXIst century
}

\section{Celso Ladera}

Celso Luis Ladera, "Up-to-date teaching and learning of quantum optics at the beginning of the XXIst century," Proc. SPIE 4588, Seventh International Conference on Education and Training in Optics and Photonics, (28 May 2002); doi: $10.1117 / 12.468742$

SPIE Event: Education and Training in Optics and Photonics 2001, 2001, Singapore, Singapore 


\title{
Up-to-date teaching and learning of quantum optics at the beginning of the XXIst century.
}

\author{
Celso L. Ladera \\ Departamento de Física, Universidad Simón Bolívar, \\ Apartado 89000, Caracas 1080A, Venezuela.
}

\begin{abstract}
The turn of the century has brought new perspectives for teaching Quantum Optics. Recent research results provide opportunities to educate specialists in the area with considerable less efforts than in the recent past. Important experiments can now be performed using cheaper optical sources. Full quantum electrodynamics approaches are often simpler to understand, and indeed more comprehensive than the semi-classical ones used before. This correlates well with the fact that it is easier to introduce quantum mechanics using Feynman's many path approach, the root of quantum electrodynamics, instead of the traditional picture based on a set of postulates. A set of cases is presented to demonstrate that full quantization of radiation and matter is not that hard to grasp by physics students with a background in quantum mechanics. The strong motivation achieved is reinforced with a set of medium cost experiments in which matter and radiation are seeing to interact, sometimes in surprising ways. Not to mention the motivating applications and hightechnology potential of present quantum optics, the teaching of both introductory and advanced quantum optics can now be performed at the highest level with an effort which, if not less, is comparable with the required when using the semiclassical approach.
\end{abstract}

Keywords: quantum optics, teaching quantum optics, full-quantization, quantum optics experiments, second quantization, quantum interference

\section{INTRODUCTION.}

This work concerns the teaching of quantum optics, an advanced topic of modern optics usually taught either to last year undergraduate electrical engineering and physics students, or definitely at the graduate level. It is therefore assumed that the subject is being presented to students who are already acquainted with modern physics and quantum mechanics. In particular it is assumed that students have mastered topics such as the quantum harmonic oscillator, and perturbation theory, and are familiar with basic spectroscopy experiments such as the Zeeman effect. Quantum optics is the theory that explains the interaction of matter and radiation, and was born in 1905 when Einstein presented his explanation of the photoelectric effect in terms of the photon. Wrongly envisioned at times as a corpuscle of light, the latter is an object that belongs to the so-called microscopic world; it is in fact the quantum object for studying light. In the days that followed, nearly 50 years, quantum optics did not show major developments. The well-known and crucial experiment of intensity correlation performed by Hanbury-Brown and Twiss ${ }^{1}$ using an intensity interferometer in 1956, the posterior appearance of the first laser $^{2}$ in 1960, and the first demonstration of non-classical light ${ }^{3}$ in 1977 consolidated quantum optics as a well-established modern physics field. A field with an stature such as that of relativity or solid state physics. Non-classical light is light with properties that essentially result from its quantum nature, and that cannot be explained using a classical physics model. By 1977 there were still doubts on the existence of the photon, it was precisely this nonclassical light experiment in 1977 that finally settled the discussion in favour of the photon theory. Scientific evidence for the photon is thus a very relevant topic for any quantum optics course.

The development of innumerable lasers in the visible, in the I.R., and in the near U.V regions of the electromagnetic (E-M) spectrum in the following 25 years, and the development or exploitation of many different optical devices since 1970, have made a reality innumerable dreamed, and undreamed, quantum optics experiments. The same can be said about a number of important applications which are the only outcome of quantum optics. One of the most attractive features of quantum optics as a teaching subject is that it offers a number of crucial experiments to test fundamental predictions made in other fields of physics (for instance in relativity). Spectacular results such as the recent demonstration of the so-called slow light ${ }^{4}$ and light-storage ${ }^{5}$ have brought quantum optics to the exhibition stages where 
the layman peers. So many developments are much too relevant to escape unnoticed to physics lecturers and departments. But the teaching of quantum optics poses a number of technical and pedagogical problems.

In spite of what has been said quantum optics is still far from being a subject ripe for its insertion in the curriculum earlier that at the senior level. Interest in the subject is growing fast however, as the publication of a significant number of important textbooks in the last ten years makes evident. From the pedagogical point of view there are a number of problems to be surmounted before quantum optics gains the terrain it deserves in the physics curriculum. The usual requirements of familiarity with electromagnetism, quantum mechanics and statistical physics are the main barriers. Quantum optics standard approach uses a model in which radiation is treated with Maxwell electromagnetic theory while matter, in interaction with the radiation, is studied using the traditional Schrödinger version of quantum mechanics. This may be called the semi-quantized approach $^{6,7}$.

Two teaching and research alternatives exists, the two of increasing sophistication, namely the fully-quantized formalism $^{7}$ (FQ) and the field quantization-matter second quantization formalism ${ }^{7}$ (FQMSQ). In the first of these approaches the E-M field waves are treated using the well-known quantized harmonic oscillator, while the interacting electron of the atom is treated quantum mechanically (i.e. similar to the electron in a hydrogen atom) and represented by its quantum state wavefunction, or matter field, $\psi$. This is the reason for naming this model fully-quantized: both radiation field and matter are quantum mechanically treated. In the more advanced approach the E-M field is again treated as a quantum harmonic oscillator, but the interacting electron is treated using the so-called second quantization. According to the latter, the matter field $\psi$ being just a wave-field can be quantized once more (in the same way that the radiation E-M field was) and thus can be seen again as true discrete quantum objects (i.e. the corpuscular nature of the electron is recovered). In the FQMSQ formalism matter (the electron) is thus seen to have underwent two quantizations: in the first of them it is represented by a quantum wavefunction field $\psi$, in the second that field $\psi$ is converted into quantum states of a discrete quantum object of definite energy. But now the operators of the new quantum model are different.

Both, from the point of view of the experiments that illustrate it, quantum optics is a demanding subject which has to be placed in the curriculum either at the final undergraduate year or definitely at the graduate level. Quantum optics experiments have always being quite sophisticated, requiring pieces of equipment of high cost not very seldom found in senior laboratories. Sources such as argon or pulsed lasers, high gain photodetection and advanced polarization devices are commonly required, adding thus to the difficulties for an earlier presentation of the subject. But there are signs of change. New powerful and low cost sources of coherent light have appeared in the scene in the last twenty years, and some of these sources are even tunable. The lower price of electrical power sources, nonlinear devices and of light detectors, plus the widespread use of computers in the laboratory, is also beginning to make a difference as far as quantum optics experiments is concerned. Moreover, after one gets familiarized with the most advanced theoretical model of Quantum Optics, that is the FQMSQ formalism, one begins to realize that it is not only more general than the two precedent theoretical models but comparatively easier to apply.

\section{FULLY QUANTIZED FORMALISMS}

The quantization of the classical E-M field is achieved ${ }^{7}$ by noting that its typical mode is a single travelling cosine plane wave of electric field $\mathrm{E}=\mathrm{E}_{0} \cos (\omega \mathrm{t}-\mathrm{kx})$ of amplitude $\mathrm{E}_{0}$, angular frequency, and $\omega$ and wavenumber $\mathrm{k}$. Magnetic field B is represented analogously. The total energy $\mathrm{U}$ in this E-M field is easily shown to be given by $\mathrm{U}=1 / 2\left(\varepsilon \mathrm{E}^{2}+\mathrm{B}^{2} / \mu\right)$. Noting the similarities with similar expressions for the harmonic oscillator, it is therefore very natural ${ }^{7}$ to quantize both fields by introducing an equivalent quantum harmonic oscillator with creation and annihilation boson operators $\mathbf{b}^{+}$and $\mathbf{b}$ respectively s.t. the Hamiltonian operator of the E-M field is simply given by $\mathbf{H}=\hbar \omega\left(\mathbf{b}^{+} \mathbf{b}+\right.$ 1/2). In the mean time the classical fields become the following field operators (where $L$ is just the length of the cavity where the field modes are defined; $N$ a normalization constant),

$$
\mathbf{E}_{z}=i N \sqrt{\frac{\hbar \omega}{\varepsilon_{0} L}}\left(\mathbf{b}^{+}-\mathbf{b}\right) \sin (k x) ; \quad \mathbf{B}_{y}=N \sqrt{\frac{\hbar \omega \mu_{0}}{L}}\left(\mathbf{b}^{+}+\mathbf{b}\right) \cos (k x) .
$$


Apart from the constants, and from the $\sin$ and cos functions which just represent the classical E-M mode spatial dependences, these field quantum operators are seen to be just the difference $\left(\mathbf{b}^{+}-\mathbf{b}\right)$ and the sum $\left(\mathbf{b}^{+}+\mathbf{b}\right)$ of the two creation and annihilation operators of the assumed quantum harmonic oscillator. In the FQ formalism, to deal with the E-M field amounts in principle to an application of the quantum harmonic oscillator formalism presented in standad quantum mechanics courses. Note that the two boson operators $\mathbf{b}^{+}$and $\mathbf{b}$ should therefore satisfy the well-known algebra of the harmonic oscillator.

Let us now consider the more sophisticated FQMSQ formalism ${ }^{7}$. The quantization of the E-M field is again done as presented above for the FQ formalism. As mentioned a second quantization of the interacting electron matter field is now be performed. Recall that electrons were historically first considered and studied as elementary microscopic "particles". Since 1925 quantum mechanics replaced that view by assigning a quantum state wave $\psi$ to represent the electron. No longer a "particle" the electron became a complex wavefunction field $\psi$. But being a field $\psi$ can be subjected to a quantization procedure totally analogous to the one applied to the E-M field. This leads to new creation and annihilation operators ladder $\mathbf{a}_{\mathbf{j}}{ }^{+}$and $\mathbf{a}_{\mathbf{j}}$ in the $\mathbf{j}$-th electron state, respectively. There are however two main differences with the case of the boson ladder operators of the standard quantum harmonic oscillator. First, one is now dealing with a complex wave field $\psi$ (instead of a real wave as happens in the E-M case); secondly electrons satisfy the Exclusion Principle (that is we must recall that they are fermions, while the photons are bosons). As in the case of the harmonic oscillator a vacuum state $\varphi_{0}$ can also be defined for the electron s.t. $\mathbf{a}_{\mathbf{j}} \varphi_{0}=0$ for any j-th state of the electron. An electron occupying the $j$-th state is simply represented by the operation $\mathbf{a}_{\mathbf{j}} \varphi_{0}$ ( i.e. an electron in the $\mathbf{j}$-th state is created from the vacuum applying the creation operator once). Since $\mathbf{a}_{\mathbf{j}}{ }^{+}$and $\mathbf{a}_{\mathbf{j}}$ are fermion ladder operators, and since the energy levels of the electrons do not correspond to harmonic oscillator energies, their algebra is different to boson algebra. For instance, for the boson ladder operators (of different wavelengths $\lambda, \lambda^{\prime}$ ) one writes the commutator $\left[\mathbf{b}_{\lambda}, \mathbf{b}^{+} \lambda^{-}\right]$ $=\delta_{\lambda \lambda^{\prime}}$, while for the fermion operators one should write instead the anti-commutator $\left\{\mathbf{a}_{\mathbf{j}}, \mathbf{a}_{\mathbf{k}}\right\}=\mathbf{a}_{\mathbf{j}} \mathbf{a}_{\mathbf{k}}+\mathbf{a}_{\mathbf{k}}, \mathbf{a}_{\mathbf{j}}=\delta_{\mathrm{jk}}$. Of course when written simultaneously in a given equation the fermion operators and boson operators commute, since they refer to different quantum objects. This is a fact that helps to simplify calculations. It happens that it is usually straightforward to write the Hamiltonian of most photon-atom interactions using the FQMSQ formalism. Since writing the proper Hamiltonian represents about half of the solution of a quantum mechanics problem, this advantage of the formalism is greatly appreciated. To proceed then to solve the resulting Schrödinger equation of the system demands extra efforts, these however are comparable to the ones when using less advanced formalisms. The advantage is the ease to write the Hamiltonian. Typical theoretical examples can be given but are out of the scope of the present work.

Three main advantages of the FQMSQ formalism have to be emphasized. The first is that it is elegant enough to allow one to tackle even many-body problems (for instance interactions between many electrons and many photons) without resorting to ad-hoc formalisms, secondly there is a perfect correlation with the useful Feynman diagrams formalism (Fig. 1), and finally it is only within this more general formalism that the whole realm of quantum optics phenomena are fully explained (e.g. the quantum mechanical Langevin equations for a set of field modes in interaction with a system of many level atoms).

\section{A SET OF AVAILABLE EXPERIMENTS}

Below a list is presented of some undergraduate experiments which are low or medium-to-high cost, and that reveal the quantum nature of light, as well as the need for fully-quantized models. Some of them are striking experiments indeed and add to the arguments in favour of the teaching of the fully-quantized formalisms of quantum optics instead of the usual semi-quantized approah. The list is by no means exhaustive, it is only presented as a true sample of what can be done. In fact most of them have been recently presented as experiments to be introduced in senior student laboratories.

\subsection{The discrete nature of photons.}

The lumpy nature of photons is usually inferred from the photo-electric effect experiment performed at the undergraduate freshmen or junior physics laboratory. But by measuring the minimum potential differences of LEDs of different colour one gets evidence for Einstein-Planck photon energy relation $(\mathrm{E}=\mathrm{hv})$. At a more sophisticated level the detection of gamma radiation, or rather the random arrival of gamma photons at the detector reveals not only the lumpy nature of photons but also their random emission from the decaying atoms. 


\subsection{An interesting gedanken experiment.}

The superposition of two parallel and equally intense collimated coherent beams of light incident on a given surface is an interesting gedanken experiment. A paradoxical violation of the conservation of energy results when one applies the well-known modulus-squared of the total incident amplitude to evaluate the energy flux: one gets either zero or four times the expected energy flux. This experiment quickly carries one, in a highly motivating way, to the problem of interfering photons and correlation experiments.

\subsection{Photon counting statistics.}

Both the statistical fluctuations of light and the probabilistic nature of the photodetection process (for both coherent and pseudo-thermal light) can be demonstrated ${ }^{8}$ using an ordinary continuous wave laser, a photomultiplier and a rotating plane scattering surface, plus a personal computer.

\subsection{Non-classical light.}

A nice experiment ${ }^{9}$ that shows the quantum behaviour of light and the need for the application of fully quantized formalisms can be performed with a LED, photodiodes and an spectrum analyzer. The results of the experiments violates both the predictions made by the classical and semi-classical models of light.

\subsection{Dark states in coherent trapping.}

This is a very relevant experiment ${ }^{10}$ which can either be explained with the FQ or the FQMSQ formalisms, the second one offering more complete information ${ }^{11}$. The experiment is performed with two laser beams that simultaneously excite an atomic vapour, in the presence of an external magnetic field. It is a nice quantum interference experiment, in fact a macroscopic quantum effect, i.e. one that occurs at the microscopic level but that can be directly seen by the observer at the macroscopic level.

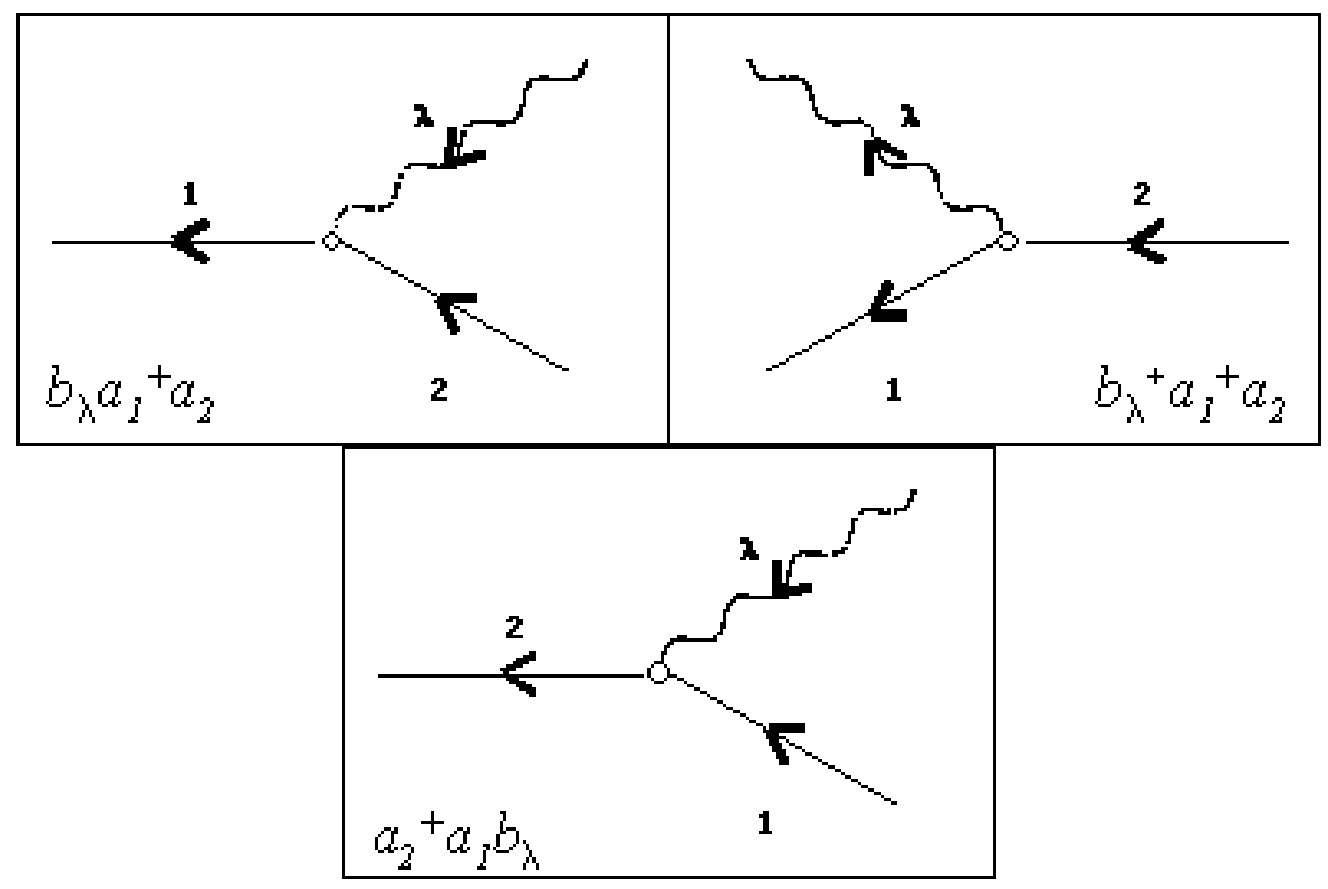

Fig. 1. FQMSQ formalism and Feynman diagrams. Photons are represented as wavy lines, electron states as straight arrows. The top-left diagram is interpreted as follows: a photon of wavelength $\lambda$ is destroyed (the photon annihilation operator $b_{\lambda}$ is applied), an electron in state 1 disappears (fermion annihilation $\mathrm{a}_{1}$ is applied), while an electron in state 2 is created. (fermion creation operator $\mathrm{a}_{2}$ ). The final arrow, denoted 2, represents the electron in the final state 2 . The rest of the diagrams are similarly interpreted. 


\section{REFERENCES}

1. R. Hanbury-Brown and R. Q. Twiss, "Correlation between photons in two coherent beams of light", Nature 127, 27-30 (1956).

2. T. H. Maiman, "Stimulated optical radiation in ruby", Nature 187, 493-494 (1960).

3. H. J. Kimble, M. Dagenais and L. Mandel, Phys. Rev. Latt. 56, 58-62 (1977).

4. L. V. Hau, S. E. Harris, Z. Dutton and C. H. Behroozi, "Light speed reduction 17 meters per second in an ultracold atomic gas", Nature 594-598 (1999).

5. D. F. Phillips, A. Fleischauer, A. Mair, R. L. Waldsworth and M. D. Lukin, "Storage of light in atomic vapor", Phys. Rev. Lett., 783-786 (2001).

6. H. Haken, "The Semi-Classical and Quantum Theory of the Laser", in Quantum Optics, p. 201-321, S. M. Kay and A. Maitland (Eds), Academic Press, London (1970).

7. H. Haken, Light: waves, atoms and photons 1, Ch. 7-9, North-Holland, Amsterdam (1981).

8. P. Koczyk, P. Wiewor and C. Radzewics, "Photon-counting statistics-Undergraduate experiment", Am. J. Phys., 64, 240-245 (1996).

9. A. C. Funk and M. Beck, "Sub-poissonian photocurrent statistics: theory and undergraduate experiment", Am. J. Phys., 65 492-500 (1997).

10. G. Alzetta, A. Gozzini, L. Moi and G. Orriols, "An experimental method for the observation of RF transitions and laser beat resonances in oriented Na vapour", Nuovo Cimento, 36B, 5-20 (1976).

11. P. A. Pineda, Physics Degree Thesis (C. L. Ladera, Academic Supervisor), Physics Department, Universidad Simón Bolívar, Caracas (2001). 